\title{
Decentralized Disturbance Attenuation for Large-Scale Nonlinear Systems with Delayed State Interconnections
}

\author{
Yi Guo
}

\begin{abstract}
The problem of decentralized disturbance attenuation is considered for a new class of large-scale nonlinear systems with delayed state interconnections. This class of largescale time-delay systems broadens most existing classes of large-scale time-delay systems in that the uncertain interconnections are bounded by general nonlinear functions instead of linear or polynomial-type functions. It is shown that by decentralized memoryless state feedback control, the closedloop system achieves internal global asymptotical stability in the sense of Lyapunov and external stability in the sense of $\mathcal{L}_{2}$ gain. Nonlinear Lyapunov-Krasovskii functionals are constructed which renders the linear and polynomial-type growth conditions on the interconnections as special cases.
\end{abstract}

Keywords: Large-scale systems, decentralized control, timedelay, nonlinear control, backstepping.

\section{INTRODUCTION}

Decentralized control has received renewed interests during the past years, motivated by its importance in applications to large complex engineering systems. Advances in robust control and stability analysis for centralized systems have been extended to large-scale interconnected systems. The existence of time delay is frequently a source of instability, see [1], [2]. Among many contributions on time-delay systems (see [3] for a review), robust $H_{\infty}$ control for linear state-delayed systems were studied in [4] using the linear matrix inequality method, and in [5] using backstepping for systems with delay in control. Decentralized control for large-scale time-delay interconnected systems has also received considerable attention. In [6], [7], decentralized stabilization for linear time-invariant large-scale systems with time-delay was discussed. Large-scale linear timevarying systems with delayed state perturbations in the interconnections were considered in [8]. The authors in [9], [10] consider the decentralized stabilization for nonlinear large-scale systems including state delays in the interconnections, where the interconnections are assumed to be bounded by linear functions of states and/or delayed states with matching conditions. It seems that for uncertain largescale time-delay interconnected systems, decentralized control results for strong interconnections beyond linear bounds have not been reported yet in the control literatures.

In this paper, we extend our earlier result in [11] to large-scale nonlinear systems with delayed state interconnections. The class of large-scale time-delay systems under consideration significantly broadens most existing classes

Yi Guo is with the Department of Electrical and Computer Engineering, University of Central Florida, P.O. Box 162450, Orlando, FL 328162450, USA. Phone: +1407 8235762. Fax: +1407 8235835. Email: yguo@ee.ucf.edu. of large-scale time-delay systems. Combining the recent development in decentralized nonlinear control, we consider a class of nonlinear systems in their decentralized diagonal form. We design decentralized memoryless state feedback controllers to solve the so-called "Problem of decentralized $H_{\infty}$ almost disturbance decoupling". On the basis of dissipativity/Lyapunov-stability theory and LyapunovKrasovskii functional, we prove the asymptotical stability for the interconnected systems in the absence of disturbance; and in the case of disturbance attenuation, any given $\mathcal{L}_{2}$ gain from the disturbance to the system output is obtained. The notation used in this paper is standard and is the same as in [11].

\section{Problem Formulation}

We consider in this paper a class of large-scale time-delay nonlinear systems $S$ which are composed of single-input single-output (SISO) subsystems $S_{i}(1 \leq$ $i \leq N)$ as described in (1), where $z_{i}(t)=$ $\left[z_{i 1}(t), \ldots, z_{i \kappa}(t)\right] \in \Re^{\kappa}, z(t)=\left[z_{1}^{T}(t), \ldots, z_{N}^{T}(t)\right]^{T}$, $\xi_{i}(t)=\left[\xi_{i 1}(t), \ldots, \xi_{i, n-\kappa}(t)\right] \in \Re^{n-\kappa}$, and $\xi(t)=$ $\left[\xi_{1}^{T}(t), \ldots, \xi_{N}^{T}(t)\right]^{T}, z(t)$ and $\xi(t)$ are the state vectors, $u_{i}(t) \in \Re$ is the control input, $\omega_{i}(t) \in \Re^{m_{i}}$ is the disturbance input, $y_{i}(t) \in \Re$ is the to-be-controlled output; the unknown functions $\gamma_{i l}, p_{i l}, \quad(0 \leq l \leq n-\kappa)$ are locally Lipschitz in states and piecewise continuous in $t$, and $\gamma_{i l}(t, 0,0, \ldots, 0)=0 ; h_{i j}(i, j=1,2, \ldots, N)$ are the time delays; $A_{i}, B_{i}$ are constant matrices and

$$
A_{i}=\left[\begin{array}{cc}
0 & \\
\vdots & I_{(\kappa-1) \times(\kappa-1)} \\
0 & 0 \ldots 0
\end{array}\right], B_{i}=\left[\begin{array}{c}
0_{(\kappa-1) \times 1} \\
1
\end{array}\right] .
$$

The initial condition for $z_{i}$-subsystem with time delays is given by

$$
z_{i}\left(t_{0}\right)=\chi_{i}\left(t_{0}\right), \quad t_{0} \in\left[-h_{i}, 0\right]
$$

where $\chi_{i}\left(t_{0}\right)$ is a uniformly continuous function on $\left[-h_{i}, 0\right]$, and $h_{i}$ is defined as: $h_{i}=\max \left\{h_{i j}, j=1,2, \ldots, N\right\}$.

Remark 1: The class of systems (1) has been motivated by several early papers in the nonlinear control literature and also exists in large power systems (see [12], [13]). Necessary and sufficient geometric conditions are given for the non-delay time-invariant decentralized strict feedback form in [14]. For systems having nonlinear zero dynamics, similar results in the argument of integral-input-to-state stability using output feedback were obtained in [15]. It 


$$
\begin{gathered}
\dot{z}_{i}(t)=A_{i} z_{i}(t)+B_{i}\left[\xi_{i 1}(t)+\gamma_{i 0}\left(t, z_{1}\left(t-h_{i 1}\right), \ldots, z_{N}\left(t-h_{i N}\right)\right)+p_{i 0}\left(t, z_{1}\left(t-h_{i 1}\right), \ldots, z_{N}\left(t-h_{i N}\right)\right) \omega_{i}(t)\right] \\
\dot{\xi}_{i 1}(t)=\xi_{i 2}(t)+\gamma_{i 1}\left(t, z_{1}\left(t-h_{i 1}\right), \ldots, z_{N}\left(t-h_{i N}\right), \xi_{i 1}(t)\right)+p_{i 1}\left(t, z_{1}\left(t-h_{i 1}\right), \ldots, z_{N}\left(t-h_{i N}\right), \xi_{i 1}(t)\right) \omega_{i}(t) \\
\dot{\xi}_{i 2}(t)=\xi_{i 3}(t)+\gamma_{i 2}\left(t, z_{1}\left(t-h_{i 1}\right), \ldots, z_{N}\left(t-h_{i N}\right), \xi_{i 1}(t), \xi_{i 2}(t)\right)+p_{i 2}\left(t, z_{1}\left(t-h_{i 1}\right), \ldots, z_{N}\left(t-h_{i N}\right), \xi_{i 1}(t), \xi_{i 2}(t)\right) \omega_{i}(t) \\
\vdots \\
\dot{\xi}_{i, n-\kappa}(t)=u_{i}(t)+\gamma_{i, n-\kappa}\left(t, z_{1}\left(t-h_{i 1}\right), \ldots, z_{N}\left(t-h_{i N}\right), \xi_{i}(t)\right)+p_{i, n-\kappa}\left(t, z_{1}\left(t-h_{i 1}\right), \ldots, z_{N}\left(t-h_{i N}\right), \xi_{i}(t)\right) \omega_{i}(t) \\
y_{i}(t)=z_{i 1}(t)
\end{gathered}
$$

is worth noting that the structure of the interconnections are broadened in (1) by: 1) removing the strict matching conditions for all local and interconnecting uncertainties as in [16], [9], [10]; 2) removing the linear or polynomial-type growth conditions on the interconnections imposed in [14], [9], [10].

The following assumptions will be used to restrict our systems (1):

Assumption 1: There exist known smooth functions $a_{i l j}(\cdot), b_{i l j}(\cdot), \varphi_{i l j}(\cdot), \Phi_{i l j}(\cdot)$, with $\varphi_{i l j}(0)=0, \Phi_{i l j}(0)=$ 0 , such that for each $0 \leq l \leq n-\kappa$ the uncertain interconnections satisfy

$$
\begin{aligned}
& \mid \gamma_{i l}\left(t, z_{1}\left(t-h_{i 1}\right), \ldots, z_{N}\left(t-h_{i N}\right), \xi_{i 1}(t), \ldots, \xi_{i l}(t)\right) \\
& -\gamma_{i l}\left(t, 0, \ldots, 0, \xi_{i 1}(t), \ldots, \xi_{i l}(t)\right) \mid \\
\leq & \sum_{j=1}^{N} a_{i l j}\left(\xi_{i 1}(t), \ldots, \xi_{i l}(t)\right) \varphi_{i l j}\left(\left|z_{j}\left(t-h_{i j}\right)\right|\right), \\
& \mid p_{i l}\left(t, z_{1}\left(t-h_{i 1}\right), \ldots, z_{N}\left(t-h_{i N}\right), \xi_{i 1}(t), \ldots, \xi_{i l}(t)\right) \\
& -p_{i l}\left(t, 0, \ldots, 0, \xi_{i 1}(t), \ldots, \xi_{i l}(t)\right) \mid \\
\leq & \sum_{j=1}^{N} b_{i l j}\left(\xi_{i 1}(t), \ldots, \xi_{i l}(t)\right) \Phi_{i l j}\left(\left|z_{j}\left(t-h_{i j}\right)\right|\right) .
\end{aligned}
$$

For the sake of simplicity, we let $a_{i 0 j}=b_{i 0 j}=1$.

Assumption 2: There exist known smooth functions $\psi_{i l}(\cdot), \Psi_{i l}(\cdot)$, with $\psi_{i l}(0)=0$, such that for each $0 \leq l \leq$ $n-\kappa$,

$$
\begin{aligned}
& \left|\gamma_{i l}\left(t, 0, \ldots, 0, \xi_{i 1}(t), \ldots, \xi_{i l}(t)\right)\right| \\
\leq & \psi_{i l}\left(\left|\left(\xi_{i 1}(t), \ldots, \xi_{i l}(t)\right)\right|\right), \\
& \left|p_{i l}\left(t, 0, \ldots, 0, \xi_{i 1}(t), \ldots, \xi_{i l}(t)\right)\right| \\
\leq & \Psi_{i l}\left(\left|\left(\xi_{i 1}(t), \ldots, \xi_{i l}(t)\right)\right|\right) .
\end{aligned}
$$

Since $\gamma_{i 0}(t, 0)=0$, we can take $\psi_{i 0} \equiv 0$; and $\Psi_{i 0}$ is a nonnegative constant.

We define our control problem as the following:

Problem of Decentralized $H_{\infty}$ Almost Disturbance Decoupling: Find decentralized smooth memoryless state feedback controllers $u_{i}(t)=u_{i}\left(z_{i}(t), \xi_{i}(t)\right)$ such that, for any given positive constant $\mu$, the closed-loop interconnected system satisfies the following dissipation inequality

$$
\int_{0}^{T}|y(t)|^{2} d t \leq \mu \int_{0}^{T}|\omega(t)|^{2} d t+\nu\left(\chi\left(t_{0}\right), \xi(0)\right),
$$

$$
\forall \omega \in \mathcal{L}_{2}(0, T), \forall T \geq 0
$$

where $\nu$ is a positive semidefinite function, $\left(\chi\left(t_{0}\right), \xi(0)\right)$ denotes the initial conditions and

$$
\chi\left(t_{0}\right)=\left[\chi_{1}^{T}\left(t_{0}\right), \chi_{2}^{T}\left(t_{0}\right), \ldots, \chi_{N}^{T}\left(t_{0}\right)\right]^{T}
$$

with $\chi_{i}\left(t_{0}\right), i=1,2, \ldots, N$ are given in (3). Furthermore, the origin is globally uniformly asymptotically stable (GUAS) if $\omega=0$.

\section{Decentralized State Feedback Control DESIGN}

In the following, for the ease of presentation, we denote the argument $\left(z_{1}\left(t-h_{i 1}\right), \ldots, z_{N}\left(t-h_{i N}\right)\right)$ as $z^{h}$. When omitting the arguments, $z_{i}$ and $\xi_{i}$ denote $z_{i}(t)$ and $\xi_{i}(t)$ respectively.

Step 0: We start by considering the $z_{i}(t)$-subsystem with $\xi_{i 1}(t)$ as the virtual control input (for $i=1, \ldots, N$ ). Choose the following Lyapunov-Krasovskii functional candidate:

$$
V_{0}(z)=\sum_{i=1}^{N}\left\{W_{i}\left(F_{i}\left(z_{i}\right)\right)+\sum_{j=1}^{N} \int_{t-h_{i j}}^{t} \phi_{i j}\left(\left|z_{j}(s)\right|\right) d s\right\}_{(9)}
$$

where $W_{i}(\cdot), \phi_{i j}(\cdot)$ are both smooth $\mathcal{K}_{\infty}$ function to be chosen later, $F_{i}\left(z_{i}\right)=z_{i}^{T} P_{i} z_{i}$, and $P_{i}$ is a positive definite symmetric matrix solving the algebric Riccati equation:

$$
A_{i}^{T} P_{i}+P_{i} A_{i}-2 \varepsilon_{i} P_{i} B_{i} B_{i}^{T} P_{i}+Q_{i}=0
$$

where $\varepsilon_{i}$ is a positive constant and $Q_{i}$ is a positive definite symmetric matrix.

Differentiating (9) along the solution of $z_{i}$-subsystem, and applying the interconnection bounds (4), (5) and (7), we have

$$
\begin{aligned}
\dot{V}_{0}= & \sum_{i=1}^{N} \frac{\partial W_{i}}{\partial F_{i}}\left\{2 z _ { i } ^ { T } P _ { i } \cdot \left\{A_{i} z_{i}+B_{i}\left[\xi_{i 1}\right.\right.\right. \\
& +\left(\gamma_{i 0}\left(t, z^{h}\right)-\gamma_{i 0}(t, 0)\right) \\
& \left.\left.+\left(p_{i 0}\left(t, z^{h}\right)-p_{i 0}(t, 0)\right) \omega_{i}+p_{i 0}(t, 0) \omega_{i}\right]\right\} \\
& \left.+\sum_{j=1}^{N}\left[\phi_{i j}\left(\left|z_{j}(t)\right|\right)-\phi_{i j}\left(\left|z_{j}\left(t-h_{i j}\right)\right|\right)\right]\right\}
\end{aligned}
$$


Repeatedly using the inequality $2 a b \leq a^{2}+b^{2},(a, b \in \Re)$ for the third to fifth terms in the above equation, we obtain

$$
\begin{aligned}
& \frac{\partial W_{i}}{\partial F_{i}} 2 z_{i}^{T} P_{i} B_{i}\left(\gamma_{i 0}\left(t, z^{h}\right)-\gamma_{i 0}(t, 0)\right) \\
& \leq\left|\frac{\partial W_{i}}{\partial F_{i}}\right|^{2} \cdot\left|z_{i}^{T} P_{i} B_{i}\right|^{2} \sum_{j=1}^{N} \delta_{j 0} \\
&+\sum_{j=1}^{N} \delta_{j 0}^{-1} \varphi_{i 0 j}^{2}\left(\left|z_{j}\left(t-h_{i j}\right)\right|\right) \\
& \frac{\partial W_{i}}{\partial F_{i}} 2 z_{i}^{T} P_{i} B_{i}\left(p_{i 0}\left(t, z^{h}\right)-p_{i 0}(t, 0)\right) \omega_{i} \\
& \leq \frac{1}{4 \tau_{i}^{2}}\left|\frac{\partial W_{i}}{\partial F_{i}}\right|^{4} \cdot\left|z_{i}^{T} P_{i} B_{i}\right|^{4} \sum_{j=1}^{N} \varrho_{j 0} \\
&+\sum_{j=1}^{N} \varrho_{j 0}^{-1} \Phi_{i 0 j}\left(\left|z_{j}\left(t-h_{i j}\right)\right|\right)^{4}+N \tau_{i}\left|\omega_{i}\right|^{2}, \\
& \leq \frac{\partial W_{i}}{\partial F_{i}} 2 z_{i}^{T} P_{i} B_{i} p_{i 0}(t, 0) \omega_{i} \\
& \frac{1}{d_{2 i}}\left|\frac{\partial W_{i}}{\partial F_{i}}\right|^{2}\left|z_{i}^{T} P_{i} B_{i}\right|^{2} \Psi_{i 0}^{2}+d_{2 i}\left|\omega_{i}\right|^{2}
\end{aligned}
$$

where $d_{2 i}, \delta_{j 0}, \varrho_{i 0}, \tau_{i}$ are any positive constants.

Choose our virtual control $\xi_{i 1}=\xi_{i 1}^{*}\left(z_{i}\right)$ as

$$
\begin{aligned}
\xi_{i 1}^{*}\left(z_{i}\right)= & -\left\{\varepsilon_{i} B_{i}^{T} P_{i} z_{i}+\frac{1}{2} \frac{\partial W_{i}}{\partial F_{i}}\left(z_{i}^{T} P_{i} B_{i}\right)\left(\sum_{j=1}^{N} \delta_{j 0}\right)\right. \\
& +\frac{1}{8 \tau_{i}^{2}}\left(\frac{\partial W_{i}}{\partial F_{i}}\right)^{3}\left(z_{i}^{T} P_{i} B_{i}\right)^{3}\left(\sum_{j=1}^{N} \varrho_{j 0}\right) \\
& \left.+\frac{1}{2 d_{2 i}} \frac{\partial W_{i}}{\partial F_{i}}\left(z_{i}^{T} P_{i} B_{i}\right) \Psi_{i 0}^{2}\right\}
\end{aligned}
$$

Notice $\xi_{i 1}^{*}(0)=0$.

When substituting (15) into (14), we get:

$$
\begin{aligned}
\dot{V}_{0} \leq & \sum_{i=1}^{N}\left\{\frac{\partial W_{i}}{\partial F_{i}}\left(-z_{i}^{T} Q_{i} z_{i}\right)+\sum_{j=1}^{N}\left[\phi_{i j}\left(\left|z_{j}\right|\right)\right]\right. \\
& +\sum_{j=1}^{N}\left[\delta_{i 0}^{-1} \varphi_{i 0 j}^{2}\left(\left|z_{j}\left(t-h_{i j}\right)\right|\right)\right. \\
& \left.+\varrho_{j 0}^{-1} \Phi_{i 0 j}^{4}\left(\left|z_{j}\left(t-h_{i j}\right)\right|\right)-\phi_{i j}\left(\left|z_{j}\left(t-h_{i j}\right)\right|\right)\right] \\
& \left.+\left(N \tau_{i}+d_{2 i}\right)\left|\omega_{i}\right|^{2}\right\} \\
\triangleq & \sum_{i=1}^{N}\left\{-\alpha_{i 0}\left(\left|z_{i}\right|\right)+\sum_{j=1}^{N} \beta_{i 0 j}\left(\left|z_{j}\right|\right)\right. \\
& \left.+\sum_{j=1}^{N} \beta_{i 0 j}^{h}\left(\left|z_{j}\left(t-h_{i j}\right)\right|\right)+\gamma_{i 0}\left|\omega_{i}\right|^{2}\right\}
\end{aligned}
$$

The choice of $W_{i}, \phi_{i j}$ are left till the final stage of the design problem. Note that the inequality (16) is only valid when $\xi_{i 1}=\xi_{i 1}^{*}$; otherwise, additional terms must be included. From here, we start our recursive backstepping procedure.

Step 1: Augment the $z_{i}$-subsystem with the $\xi_{i 1}$-subsystem (for $i=1, \ldots, N$ ), and choose a storage function as

$$
V_{1}\left(z, \xi_{1}\right)=V_{0}(z)+\sum_{i=1}^{N}\left(\xi_{i 1}-\xi_{i 1}^{*}\right)^{2}
$$

Denote

$$
\begin{aligned}
-\dot{\xi}_{i 1}^{*}= & -\frac{\partial \xi_{i 1}^{*}}{\partial z_{i}}\left(A_{i} z_{i}+B_{i} \xi_{i 1}\right) \\
& -\frac{\partial \xi_{i 1}^{*}}{\partial z_{i}} B_{i}\left[\gamma_{i 0}(t, z)+p_{i 0}(t, z) \omega_{i}\right] \\
\triangleq & \vartheta_{i 1}\left(z_{i}, \xi_{i 1}\right)+\sigma_{i 0}\left(z_{i}\right)\left[\gamma_{i 0}\left(t, z^{h}\right)+p_{i 0}\left(t, z^{h}\right) \omega_{i}\right]
\end{aligned}
$$

Differentiating $V_{1}\left(z, \xi_{1}\right)$ along the solutions of the $\left(z_{i}, \xi_{i 1}\right)$-subsystem yields (18), where we denote $\widetilde{\xi}_{i 1}=$ $\xi_{i 1}-\xi_{i 1}^{*}\left(z_{i}\right)$ and $\sigma_{i 1}=1$.

Since $\xi_{i 1}^{*}\left(z_{i}\right)$ is smooth and vanishes at the origin, there exists a smooth function $\widetilde{\zeta}_{i}\left(z_{i}\right)$ such that

$$
\left|\xi_{i 1}\right| \leq \widetilde{\zeta}_{i}\left(z_{i}\right) \cdot\left(\left|\widetilde{\xi}_{i 1}\right|+\left|z_{i}\right|\right)
$$

Applying the inequalities in Assumptions 1 and 2, and after some calculations, we have:

$$
\begin{aligned}
& 2 \widetilde{\xi}_{i 1} \gamma_{i 1}\left(t, 0, \xi_{i 1}\right) \\
\leq & 2\left|\widetilde{\xi}_{i 1}\right|^{2} \widetilde{\psi}_{i 1}\left(z_{i}, \xi_{i 1}\right)+\frac{1}{d_{1 i}}\left|\widetilde{\xi}_{i 1}\right|^{2} \widetilde{\psi}_{i 1}^{2}\left(z_{i}, \xi_{i 1}\right)+d_{1 i}\left|z_{i}\right|^{2} \\
& 2 \widetilde{\xi}_{i 1} \sum_{k=0}^{1} \sigma_{i k} p_{i k}\left(t, 0, \xi_{i 1}\right) \omega_{i} \\
\leq & \frac{1}{d_{2 i}}\left|\widetilde{\xi}_{i 1}\right|^{2}\left[\sum_{k=0}^{1} \sigma_{i k} \Psi_{i k}\left(\left|\xi_{i k}\right|\right)\right]^{2}+d_{2 i}\left|\omega_{i}\right|^{2} \\
& 2 \widetilde{\xi}_{i 1} \sum_{k=0}^{1} \sigma_{i k}\left[\gamma_{i k}\left(t, z^{h}, \xi_{i 1}\right)-\gamma_{i k}\left(t, 0, \xi_{i 1}\right)\right] \\
\leq & \left|\widetilde{\xi}_{i 1}\right|^{2} \sum_{k=0}^{1}\left|\sigma_{i k}\right|^{2} \sum_{j=1}^{N} \delta_{j k} a_{i k j}^{2}\left(\xi_{i 1}\right) \\
& +\sum_{k=0}^{1} \sum_{j=1}^{N} \delta_{j k}^{-1} \varphi_{i k j}^{2}\left(\left|z_{j}\left(t-h_{i j}\right)\right|\right), \\
& +\sum_{k=0}^{1} \sum_{j=1}^{N} \varrho_{j k}^{-1} \Phi_{i k j}^{4}\left(\left|z_{j}\left(t-h_{i j}\right)\right|\right)+2 N \tau_{i}\left|\omega_{i}\right|^{2} \\
& \frac{1}{4 \tau_{i}^{2}} \mid \sum_{k=0} \sigma_{i k}\left[p_{i k}\left(t, z^{h}, \xi_{i 1}\right)-p_{i k}\left(t, 0, \xi_{i 1}\right)\right] \omega_{i} \\
& 1 \\
&
\end{aligned}
$$




$$
\begin{gathered}
\dot{V}_{1} \leq \sum_{i=1}^{N}\left\{-\alpha_{i 0}\left(\left|z_{i}\right|\right)+\sum_{j=1}^{N} \beta_{i 0 j}\left(\left|z_{j}\right|\right)+\sum_{j=1}^{N} \beta_{i 0 j}^{h}\left(\left|z_{j}\left(t-h_{i j}\right)\right|\right)+\gamma_{i 0}\left|\omega_{i}\right|^{2}+2 \widetilde{\xi}_{i 1}\left\{\xi_{i 2}+\left(\frac{\partial W_{i}}{\partial F_{i}} z_{i}^{T} P_{i} B_{i}+\vartheta_{i 1}\right)\right.\right. \\
\left.\left.+\sum_{k=0}^{1} \sigma_{i k}\left[\gamma_{i k}\left(t, z^{h}, \xi_{i 1}\right)-\gamma_{i k}\left(t, 0, \xi_{i 1}\right)\right]+\sum_{k=0}^{1} \sigma_{i k}\left[p_{i k}\left(t, z^{h}, \xi_{i 1}\right)-p_{i k}\left(t, 0, \xi_{i 1}\right)\right] \omega_{i}+\gamma_{i 1}\left(t, 0, \xi_{i 1}\right)+\sum_{k=0}^{1} \sigma_{i k} p_{i k}\left(t, 0, \xi_{i 1}\right) \omega_{i}\right\}\right\}
\end{gathered}
$$

where $c_{i 1}$ is a positive constant, $d_{1 i}, d_{i 2}, \delta_{i k}, \varrho_{i k}, c_{i 1}$ are positive constants, and $\widetilde{\psi}_{i 1}$ is a smooth nonnegative function.

Choose the virtual controller $\xi_{i 2}=\xi_{i 2}^{*}$ as in (24).

Substitute (20)-(23) into (18). After simplifications, we obtain

$$
\begin{aligned}
\dot{V}_{1} \leq & \sum_{i=1}^{N}\left\{-\alpha_{i 1}\left(\left|z_{i}\right|\right)-C_{i 11} \widetilde{\xi}_{i 1}^{2}+\sum_{j=1}^{N} \beta_{i 0 j}\left(\left|z_{j}\right|\right)\right. \\
& \left.+\sum_{j=1}^{N} \beta_{i 1 j}^{h}\left(\left|z_{j}\left(t-h_{i j}\right)\right|\right)+\gamma_{i 1}\left|\omega_{i}\right|^{2}\right\}
\end{aligned}
$$

where $\alpha_{i 1}\left(\left|z_{i}\right|\right), \beta_{i 1 j}^{h}\left(\left|z_{j}\left(t-h_{i j}\right)\right|\right), \gamma_{i 1}, C_{i 11}$ are appropriate functions and constants.

Note that the inequality (25) is only valid when $\xi_{i 2}=\xi_{i 2}^{*}$, as in the standard backstepping design process.

From Step 2, we can establish the following Claim. The proof of the Claim is not difficult using the same approach as in [11], and is omitted here due to space limitations.

Claim: Given any index $2 \leq \iota \leq n-\kappa$, for the system $S_{i \iota}, i=1, \ldots, N$

$$
\begin{aligned}
\dot{z}_{i}= & A_{i} z_{i}+B_{i}\left[\xi_{i 1}+\gamma_{i 0}\left(t, z^{h}\right)+p_{i 0}\left(t, z^{h}\right) \omega_{i}\right] \\
\dot{\xi}_{i 1}= & \xi_{i 2}+\gamma_{i 1}\left(t, z^{h}, \xi_{i 1}\right)+p_{i 1}\left(t, z^{h}, \xi_{i 1}\right) \omega_{i} \\
\vdots & \\
\dot{\xi}_{i \iota}= & \xi_{i, \iota+1}+\gamma_{i \iota}\left(t, z^{h}, \xi_{i 1}, \ldots, \xi_{i \iota}\right) \\
& +p_{i \iota}\left(t, z^{h}, \xi_{i 1}, \ldots, \xi_{i \iota}\right) \omega_{i}
\end{aligned}
$$

there exist $\iota+1$ smooth functions

$$
\begin{gathered}
\xi_{i k}^{*}=\xi_{i k}^{*}\left(z_{i}, \xi_{i 1}, \ldots, \xi_{i, k-1}\right), \\
\xi_{i k}^{*}(0,0, \ldots, 0)=0,1 \leq k \leq \iota+1
\end{gathered}
$$

such that in new coordinates

$$
\begin{gathered}
\widetilde{z}_{i}=z_{i}, \widetilde{\xi}_{i k}=\xi_{i k}-\xi_{i k}^{*}\left(z_{i}, \xi_{i 1}, \ldots, \xi_{i, k-1}\right), \\
1 \leq k \leq \iota
\end{gathered}
$$

the storage function

$$
V_{\iota}=V_{0}+\sum_{i=1}^{N} \sum_{k=1}^{\iota} \widetilde{\xi}_{i k}^{2}
$$

has time derivative, with $\xi_{i, \iota+1}=\xi_{i, \iota+1}^{*}$, satisfying the dissipation inequality

$$
\begin{aligned}
\dot{V}_{\iota} \leq & \sum_{i=1}^{N}\left\{-\alpha_{i \iota}\left(\left|z_{i}\right|\right)-\sum_{k=1}^{\iota} C_{i \iota k} \widetilde{\xi}_{i k}^{2}+\sum_{j=1}^{N}\left[\beta_{i 0 j}\left(\left|z_{j}\right|\right)\right.\right. \\
& \left.\left.+\beta_{i \iota j}^{h}\left(\left|z_{j}\left(t-h_{i j}\right)\right|\right)\right]+\gamma_{i \iota}\left|\omega_{i}\right|^{2}\right\}
\end{aligned}
$$

where

$$
\begin{aligned}
& \alpha_{i \iota}\left(\left|z_{i}\right|\right)=\alpha_{i, \iota-1}\left(\left|z_{i}\right|\right)-d_{1 i}\left|z_{i}\right|^{2}, \\
& \beta_{i \iota j}^{h}\left(\left|z_{j}\left(t-h_{i j}\right)\right|\right)=\beta_{i, \iota-1, j}\left(\left|z_{j}\left(t-h_{i j}\right)\right|\right) \\
& +\sum_{k=0}^{\iota}\left\{\delta_{j k}^{-1} \varphi_{i k j}^{2}\left(\left|z_{j}\left(t-h_{i j}\right)\right|\right)\right. \\
& \left.+\varrho_{j k}^{-1} \Phi_{i k j}^{4}\left(\left|z_{j}\left(t-h_{i j}\right)\right|\right)\right\}, \\
& \gamma_{i \iota}=\gamma_{i, \iota-1}+(\iota+1) N \tau_{i}+d_{2 i}, \\
& C_{i \iota k}=\left\{\begin{array}{ccc}
C_{i, \iota-1, k}-d_{3 i} & \text { for } & k=1, \ldots, \iota-1 \\
c_{i k} & \text { for } & k=\iota
\end{array}\right.
\end{aligned}
$$

\section{MAIN Result}

The following theorem states our main result:

Theorem 1: Under Assumptions 1 and 2, the Problem of Decentralized $H_{\infty}$ Almost Disturbance Decoupling is solvable for system (1).

Proof: As shown by the Claim, at the last step, a decentralized memoryless state feedback control

$$
u_{i}(t)=\xi_{i, n-\kappa+1}^{*}\left(z_{i}(t), \xi_{i}(t)\right)
$$

is iteratively built. For the closed-loop system $S$, for $V=$ $V_{n-\kappa}$, we have

$$
\begin{aligned}
\dot{V} \leq & \sum_{i=1}^{N}\left\{-\alpha_{i}\left(\left|z_{i}\right|\right)-\sum_{l=1}^{n-\kappa} C_{i l} \widetilde{\xi}_{i l}^{2}\right. \\
& \left.+\sum_{j=1}^{N}\left[\beta_{i 0 j}\left(\left|z_{j}\right|\right)+\beta_{i j}^{h}\left(\left|z_{j}\left(t-h_{i j}\right)\right|\right)\right]+\gamma_{i}\left|\omega_{i}\right|^{2}\right\}
\end{aligned}
$$

To eliminate the term $\beta_{i j}^{h}\left(\left|z_{j}\left(t-h_{i j}\right)\right|\right)$, we choose the design function $\phi_{i j}$ in (9) as:

$$
\begin{aligned}
\phi_{i j}(|s|)= & \sum_{l=0}^{n-\kappa}\left\{(n-\kappa-l+1) \delta_{j l}^{-1} \varphi_{i l j}^{2}(|s|)\right. \\
& \left.+(n-\kappa-l+1) \varrho_{j l}^{-1} \Phi_{i l j}^{4}(|s|)\right\}
\end{aligned}
$$




$$
\begin{gathered}
\xi_{i 2}^{*}=-\frac{1}{2}\left\{c_{i 1} \widetilde{\xi}_{i 1}+\frac{\partial W_{i}}{\partial F_{i}} 2 z_{i}^{T} P_{i} B_{i}+2 \vartheta_{i 1}+2 \widetilde{\xi}_{i 1} \widetilde{\psi}_{i 1}\left(z_{i}, \xi_{i 1}\right)+\frac{1}{d_{1 i}} \widetilde{\xi}_{i 1} \widetilde{\psi}_{i 1}^{2}\left(z_{i}, \xi_{i 1}\right)\right. \\
\left.+\frac{1}{d_{2 i}} \widetilde{\xi}_{i 1}\left[\sum_{k=0}^{1} \sigma_{i k} \Psi_{i 1}\left(\left|\xi_{i k}\right|\right)\right]^{2}+\widetilde{\xi}_{i 1} \sum_{k=0}^{1} \sigma_{i k}^{2} \sum_{j=1}^{N} \delta_{j k} a_{i k j}^{2}\left(\xi_{i 1}\right)+\frac{1}{4 \tau_{i}^{2}} \widetilde{\xi}_{i 1}^{3} \sum_{k=0}^{1} \sigma_{i k}^{4} \sum_{j=1}^{N} \varrho_{j k} b_{i k j}^{4}\left(\xi_{i 1}\right)\right\}
\end{gathered}
$$

Since $\varphi_{i l j}(\cdot), \Phi_{i l j}(\cdot)$ are smooth functions and vanish at origin, there exists smooth nondecreasing functions $\widetilde{\varphi}_{j l i}\left(\left|z_{i}\right|\right), \widetilde{\Phi}_{j l i}\left(\left|z_{i}\right|\right)$ such that

$$
\begin{aligned}
& \varphi_{j l i}\left(\left|z_{i}\right|\right) \leq\left|z_{i}\right| \widetilde{\varphi}_{j l i}\left(\left|z_{i}\right|\right), \\
& \Phi_{j l i}\left(\left|z_{i}\right|\right) \leq\left|z_{i}\right| \widetilde{\Phi}_{j l i}\left(\left|z_{i}\right|\right) .
\end{aligned}
$$

Now we can choose our design function $W_{i}$ in (15). Notice that

$$
\left|z_{i}\right| \leq \sqrt{\frac{F_{i}\left(z_{i}\right)+1}{\lambda_{\min }\left(P_{i}\right)}} \triangleq \eta_{i}\left(F_{i}\right)
$$

where the functions $\eta_{i}$ are smooth positive functions.

Construct the derivative of $W_{i}$ as

$$
\begin{gathered}
\frac{\partial W_{i}}{\partial F_{i}}=k_{i}+\frac{1}{\lambda_{\min }\left(Q_{i}\right)} \sum_{j=1}^{N} \sum_{l=0}^{n-\kappa}(n-\kappa-l+1) \delta_{i l}^{-1} \\
\cdot\left[\widetilde{\varphi}_{j l i} \circ \eta_{i}\left(F_{i}\right)\right]^{2}+\frac{F_{i}}{\lambda_{\min }\left(Q_{i}\right) \cdot \lambda_{\min }\left(P_{i}\right)} \\
\cdot \sum_{j=1}^{N} \sum_{l=0}^{n-\kappa}(n-\kappa-l+1) \varrho_{i l}^{-1}\left[\widetilde{\Phi}_{j l i} \circ \eta_{i}\left(F_{i}\right)\right]^{4}
\end{gathered}
$$

where $k_{i} s$ are positive constants. It can be checked that by the above construction and $W_{i}(0)=0, W_{i}$ is a smooth $\mathcal{K}_{\infty}$ function.

In view of the above, we can check that

$$
\begin{aligned}
\sum_{i=1}^{N} \frac{\partial W_{i}}{\partial F_{i}} z_{i}^{T} Q_{i} z_{i} \geq & \sum_{i=1}^{N} k_{i} \lambda_{\min }\left(Q_{i}\right)\left|z_{i}\right|^{2} \\
& +\sum_{i=1}^{N} \sum_{j=1}^{N} \beta_{i 0 j}\left(\left|z_{j}\right|\right)
\end{aligned}
$$

Therefore, we obtain

$$
\sum_{i=1}^{N}\left\{-\alpha_{i}\left(\left|z_{i}\right|\right)+\sum_{j=1}^{N} \beta_{i 0 j}\left(\left|z_{j}\right|\right)\right\} \leq \sum_{i=1}^{N}\left(-D_{i}\left|z_{i}\right|^{2}\right)
$$

where $D_{i}=k_{i} \lambda_{\min }\left(Q_{i}\right)-(n-\kappa) d_{1 i}$. Choose the design parameter $d_{1 i}$ as

$$
d_{1 i}<\frac{k_{i} \lambda_{\min }\left(Q_{i}\right)}{n-\kappa}
$$

so that $D_{i}>0$.

Choose the design parameter $d_{3 i}$ as

$$
d_{3 i}<\min _{1 \leq l \leq n-\kappa-1}\left\{\frac{c_{i l}}{n-\kappa-l}\right\}
$$

so that $C_{i l}>0$.

Therefore we obtain:

$$
\dot{V} \leq \sum_{i=1}^{N}\left\{-D_{i}\left|z_{i}\right|^{2}-\sum_{l=1}^{n-\kappa} C_{i l} \widetilde{\xi}_{i l}^{2}+\gamma_{i}\left|\omega_{i}\right|^{2}\right\},
$$

and it follows

$$
\dot{V} \leq \sum_{i=1}^{N}\left\{-D_{i}\left|z_{i 1}\right|^{2}+\gamma_{i}\left|\omega_{i}\right|^{2}\right\} .
$$

Taking the integral along time $t$, the $\mathcal{L}_{2}$ gain from $\omega$ to $y$ of the closed-loop system is obtained as

$$
\int_{0}^{T}|y(t)|^{2} d t \leq \mu \int_{0}^{T}|w(t)|^{2} d t+\nu\left(z\left(t_{0}\right), \xi(0)\right)
$$

where

$$
\begin{gathered}
\mu=\min _{1 \leq i \leq N}\left\{\gamma_{i}\right\} / \max _{1 \leq i \leq N}\left\{D_{i}\right\}, \\
\nu\left(z\left(t_{0}\right), \xi(0)\right)=\left\{\sum_{i=1}^{N} z_{i}(0)^{T} P_{i} z_{i}(0)\right. \\
\left.+\sum_{j=1}^{N} \int_{-h_{i j}}^{0} \phi_{i j}\left(\left|z_{j}(s)\right|\right) d s+\sum_{l=1}^{n-\kappa} \widetilde{\xi}_{i l}^{2}(0)\right\} / \max _{1 \leq i \leq N}\left\{D_{i}\right\} .
\end{gathered}
$$

In the absence of the disturbance, i.e. when $\omega_{i}=0$, from (44), we have

$$
\dot{V} \leq \sum_{i=1}^{N}\left\{-D_{i}\left|z_{i}\right|^{2}-\sum_{l=1}^{n-\kappa} C_{i l} \widetilde{\xi}_{i l}^{2}\right\} \triangleq-V_{a}(z, \xi) \leq 0
$$

From (9) and (27), $V(z, \xi)$ is a continuously differentiable, positive definite and radially unbounded function and its derivative is negative definite. From the Lyapunov stability theorems for equations with bounded delays ([1, page 103]), we obtain the GUAS of the closed-loop system when $\omega_{i}=0$.

Remark 2: As an extension to the result in [11], the nett energy dissipation for the interconnected system $S$ is obtained by constructing decentralized memoryless state feedback control laws to dominate other subsystem interactions by the subsystem stability margins. This is done by choosing the Lyapunov/storage function as the sum of subsystem Lyapunov-Krasovskii functionals. The novelty of handling nonlinear bounds of interconnections is the careful selection of the nonlinear storage function $W_{i}$, as adopted in [11], [16]. The results render the linear ([6], [8], [7]) and polynomial-type ([14]) growth conditions on the 


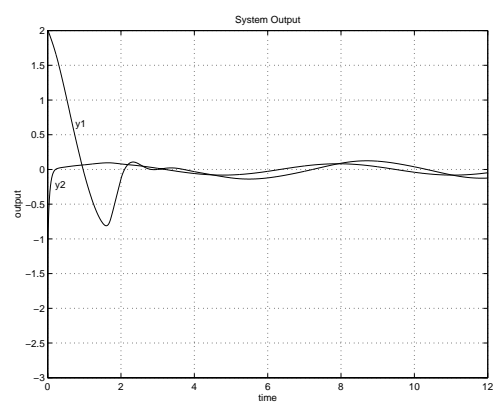

Fig. 1. Output of the closed-loop system

interconnections as special cases where $W_{i}$ is chosen to be linear and higher-order polynomial respectively.

\section{An Illustrative Example}

We consider the following interconnected time-delay system composed of two subsystems:

$$
\begin{array}{r}
S_{1}:\left\{\begin{array}{l}
\dot{z}_{11}(t)=z_{12}(t) \\
\dot{z}_{12}(t)=\xi_{11}(t)+\sin (2 t) z_{21}(t-2.0) \\
\cdot e^{\left(z_{21}(t-2.0)\right.}+\left(1+z_{11}(t-1.0) z_{21}(t)\right) \omega_{1}(t) \\
\dot{\xi}_{11}(t)=u_{1}(t)+\xi_{11}(t) z_{21}(t) \omega_{1}(t) \\
y_{1}(t)=z_{11}(t)
\end{array}\right. \\
S_{2}:\left\{\begin{array}{l}
\dot{z}_{21}(t)=\xi_{21}(t)+z_{11}(t) z_{12}(t-1.0) \sin \left(z_{21}(t)\right) \\
+\omega_{2}(t) \\
\dot{\xi}_{21}(t)=u_{2}(t)+\omega_{2}(t) \\
y_{2}(t)=z_{21}(t)
\end{array}\right.
\end{array}
$$

The initial conditions for the two subsystems are as follows:

$$
\begin{aligned}
& {\left[z_{11}(t), z_{12}(t), \xi_{11}(t)\right]^{T}=[2.0,0.5,1.0]^{T}, t \in[-2.0,0]} \\
& {\left[z_{21}(t), \xi_{21}(t)\right]^{T}=[-3.0,1.7]^{T}, t \in[-2.0,0] .}
\end{aligned}
$$

It can be checked that system $S$ is in form (1) and satisfies Assumptions 1 and 2 . We choose the design parameters $\varepsilon_{1}=1, \varepsilon_{2}=1, Q_{1}=2 I, Q_{2}=2$ to get

$$
P_{1}=\left[\begin{array}{ll}
2.8284 & 1.0000 \\
1.0000 & 1.4142
\end{array}\right], \quad P_{2}=1 .
$$

Other parameters are chosen as follows, for $i, l=1,2$,

$$
k_{i}=8, \quad \delta_{i l}=\varrho_{i l}=0.1, \quad \tau_{i}=1, \quad c_{i}=0.1, \quad d_{2 i}=2 .
$$

Construct the control law following that described in Steps 0 and 1 in Section 3. The system output is shown in Figure 1 which demonstrate a satisfactory disturbance attenuation performance of our closed-loop system. The disturbances applied are $\omega_{1}=\omega_{2}=\sin (t)$.

\section{CONCLUSIONS}

We considered a class of large-scale nonlinear systems with delayed-state interconnections in this paper. The problem of decentralized $H_{\infty}$ almost disturbance decoupling has been solved for such systems. Decentralized memoryless state feedback control laws are constructed iteratively applying backstepping. The results broaden the largescale delay systems in previous literatures to nonlinear systems with uncertain interconnections to be time-varying and bounded by general nonlinear functions. Nonlinear Lyapunov-Krasovskii functionals are constructed to prove the internal global asymptotical stability in the sense of Lyapunov and external stability in the sense of $\mathcal{L}_{2}$ gain. Simulations of a two-subsystem example show a satisfactory performance. The results are expected to be applied to practical decentralized control problems of large-scale nonlinear systems with delayed state perturbations in the interconnections.

\section{REFERENCES}

[1] V. Kolmanovskii and A. Myshkis, Applied theory of functional differential equations, ser. Mathematics and Its Applications. Dordrecht: Kluwer Academic Publishers, 1992.

[2] M. Z. Manu and J. Mohammad, Time-delay system analysis, optimaization, and application. New York: AT\&T Bell Laboratories, 1987.

[3] K. Watanabe, E. Nobuyama, and A. Kojima, "Recent advances in control of time delay systems - A tutorial review," in Proceedins of the 35th IEEE Conference on Decision and Control, Kobe, Japan, December 1996, pp. 2083-2089.

[4] C. E. de Souza and X. Li, "Delay-dependent robust $H_{\infty}$ control of uncertain linear state-delayed systems," Automatica, vol. 35, pp. 1313-1321, 1999.

[5] Y. Guo, W. Zhou, and P. L. Lee, " $H_{\infty}$ control for a class of structured time-delay systems," Systems and Control Letters, vol. 45, no. 1, pp. $35-47,2002$

[6] T. N. Lee and U. L. Radovic, "Decentralized stabilization of liner continuous and discrete-time systems with delays in interconnections," IEEE Trans. Automat. Contr., vol. 33, no. 8, pp. 757-761, 1988.

[7] S. Xie, L. Xie, and C. Wen, "Decentralized $H_{\infty}$ output feedback control of interconnected time-delay systems," Proceedings of the IEEE Conference on Decision and Control, pp. 824-828, 2000.

[8] H. Wu, "Decentralized adaptive robust control for a class of largescale systems including delayed state perturbations in the interconnections," IEEE Trans. Automat. Contr., vol. 47, no. 10, pp. 17451751,2002

[9] A. S. C. Sinha, "Stabilization of large-scale non-linear infinite delay systems," Int. J. Systems Sci., vol. 21, no. 12, pp. 2679-2684, 1990.

[10] H. S. Wu, "Decentralized stabilizing state feedback controllers for a class of large-scale systems including state delays in teh interconnections," Journal of Optimization Theory and Applications, vol. 100, no. 1, pp. 59-87, 1999.

[11] Y. Guo, Z. P. Jiang, and D. J. Hill, "Decentralized robust disturbance attenuation for a class of large-scale nonlinear systems," Systems and Control Letters, vol. 37, pp. 71-85, 1999.

[12] Y. Guo, D. J. Hill, and Y. Wang, "Nonlinear decentralized control of large-scale power systems," Automatica, vol. 36, no. 9, pp. 12751289,2000

[13] S. Jain and F. Khorrami, "Robust decentralized control of power systems utilizing only swing angle measurments," Int. J. Control, vol. 66 , no. 4 , pp. 581-601, 1997.

[14] _ - "Decentralized adaptive control of a class of large-scale interconnected nonlinear systems," IEEE Trans. Automat. Contr., vol. 42, no. 2, pp. 136-154, 1997.

[15] Z. P. Jiang, D. W. Repperger, and D. J. Hill, "Decentralized nonlinear output-feedback stabilization with disturbance attenuation," IEEE Trans. Automat. Contr., vol. 46, no. 10, pp. 1623-1629, 2001.

[16] M. C. Han and Y. H. Chen, "Decentralized control design: uncertain systems with strong interconnections," Int. J. Control, vol. 61, no. 6, pp. 1363-1385, 1995. 